\title{
O RENASCIMENTO DE UM FÁRMACO: TALIDOMIDA
}

\section{Lídia Moreira Lima ${ }^{*}$, Carlos Alberto Manssour Fraga" e Eliezer J. Barreiro ${ }^{\#}$}

Departamento de Química Orgânica, Instituto de Química, Universidade Federal do Rio de Janeiro, Cidade Universitária, CP 68006, 21944-970 Rio de Janeiro - RJ

Departamento de Fármacos, Faculdade de Farmácia, Universidade Federal do Rio de Janeiro, Cidade Universitária, CP 68006, 21944-970 Rio de Janeiro - RJ

Recebido em 13/9/00; aceito em 15/12/00

THE REBIRTH OF A DRUG: THALIDOMIDE. Thalidomide, first synthesized in 1953, was widely prescribed for morning sickness of pregnant women from 1957 to 1961, when it was found to be seriously teratogenic, having caused serious birth defect. Nowadays, a quarter of a century later, it appears that it may be a miracle drug for such diseases as leprosy, AIDS, cancer and tuberculosis.

Keywords: thalidomide; rebirth of an old drug; leprosy.

\section{HISTÓRICO}

A talidomida, $[( \pm) 2-(2,6$-dioxo-3-piperidinil)- $1 H$-isoindol1,3-(2H)-diona; ou ( \pm )-ftalimidoglutarimida (1)], foi descoberta por série de fatores fortuitos e primeiramente relatada por Wilhelm Kunz, em 1953. Sua síntese foi realizada na indústria farmacêutica alemã "Chemie Grünenthal", objetivando a preparação de pequenos peptídeos utéis na produção de novos antibióticos. Entretanto, durante a rota sintética proposta inicialmente, Wilhelm Kunz isolou um produto secundário, racêmico, não peptídico (1), que foi reconhecido pelo farmacologista da "Chemie Grünenthal", Herbert Keller, como um análogo estrutural da glutetimida (2). Posteriormente, o perfil sedativo-hipnótico de (1) foi bem caracterizado ${ }^{1,2}$.

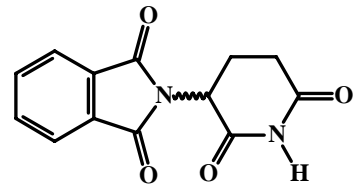

Talidomida (1) Sedativo-Hipnótico

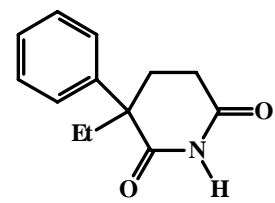

Glutetimida (2) Sedativo-Hipnótico
Em 1957, a talidomida (1) foi comercializada como fármaco sedativo-hipnótico, amplamente vendido em países europeus, asiáticos, no Canadá e América do Sul, tornando-se o medicamento mais vendido na Alemanha Ocidental para o tratamento da insônia ${ }^{3,4}$.

A despeito de sua ampla comercialização nos continentes Europeu e Asiático, este fármaco, em diferentes especialidades farmacêuticas: Kavadon ${ }^{\circledR}$, Sedalis ${ }^{\circledR}$, Softenon ${ }^{\circledR}$, Distara ${ }^{\circledR}$, Contergan ${ }^{\circledR}$, entre outros, não obteve licença do Food and Drug Administration (FDA) dos Estados Unidos da América para uso neste país ${ }^{5,6}$.

No ínicio da década de 60, a talidomida (1) foi prescrita mundialmente como sedativo e agente anti-naúseas, indicada no alívio do mal-estar matinal comum em gestantes, sendo responsável pelo nascimento de milhares de crianças com deformações congênitas, que foram, posteriormente atribuídas ao

*lidiaml@yahoo.com

\# Laboratório de Avaliação e Síntese de Substâncias Bioativas (LASSBio), http://www.farmácia.ufrj.br/lassbio. seu perfil teratogênico ${ }^{7-9}$. Após confirmação e divulgação destes graves efeitos colaterais, este fármaco teve sua licença para comercialização cancelada, rendendo, em 1961, à oficial médica americana Frances Kelsey, responsável pelas decisões à frente do FDA, a medalha e título de guardiã da Saúde Pública concedida pelo, então, presidente John Kennedy, em reconhecimento aos serviços prestados ${ }^{10,11}$.

Quatro anos mais tarde, em 1965, o médico israelita Jacob Sheskin prescreveu a talidomida (1) como sedativo para pacientes leprosos, observando fortuitamente acentuada redução da dor e do processo inflamatório associado ao leproma ${ }^{12,13}$, identificando as propriedades antiinflamatórias de (1), até então desconhecidas. Este intrigante perfil antiinflamatório incentivou diversos grupos de pesquisas ao estudo do provável mecanismo de ação relacionado às ações benéficas de (1) sobre os lepromas. Em 1991, a Dra Gilla Kaplan e colaboradores, da Universidade de Rockefeller, Nova York, em trabalho pioneiro, demonstraram que pacientes leprosos apresentavam níveis sanguíneos aumentados de um modulador do sistema imunológico, identificado como uma citocina denominada fator de necrose tumoral $\alpha(\mathrm{TNF} \alpha)^{14}$. Estes mesmos pesquisadores constataram, posteriormente, a participação da talidomida (1) na inibição seletiva desta citocina, quando expressa em quantidade superior àquela necessária à manutenção do sistema imunológico normal, denotando, portanto, o potencial imunorregulador de (1).

\section{SÍNTESE E METABOLISMO}

A metodologia sintética empregada na obtenção da $(R, S)$ talidomida (1) explorou, numa primeira etapa, a condensação do $(R, S)$-ácido glutâmico com anidrido ftálico (3), seguida da etapa chave da estratégia sintética, que consistiu na condensação do intermediário ftalimídico (4) com amônia em temperatura elevada ${ }^{15}$ (Esquema 1).

Estudos, ex vivo, sobre o destino metabólico da talidomida (1) permitiram evidenciar sua instabilidade em solução aquosa, em diferentes valores de $\mathrm{pH}$. Em $\mathrm{pH}=6,0$ observou-se a hidrólise espontânea do anel glutarimídico, originando os derivados $\alpha$-(orto-carboxi-benzamido)glutarimida (5) e (6) como principais metabólitos (Figura 1) $)^{16}$.

Entretanto, em $\mathrm{pH}$ fisiológico $(\mathrm{pH}=7,4)$ a talidomida sofre ca. $28 \%$ de metabolização na primeira hora de ensaio, sendo os principais metabólitos formados identificados como produtos de hidrólise do anel ftalimídico e glutarimídico, originando majoritariamente os compostos (7) e (8) (Figura 1) ${ }^{16}$. 
<smiles>O=C1CC(=O)c2ccccc21</smiles>

(3)

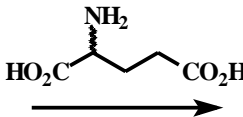<smiles>O=C(O)CCC(C(=O)O)N1C(=O)c2ccccc2C1=O</smiles>

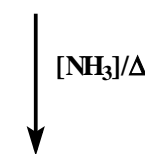<smiles>O=C1CC[C@H](N2C(=O)c3ccccc3C2=O)C(=O)N1</smiles>

Talidomida (1)
Por outro lado, em $\mathrm{pH}=8,0$ sua taxa de metabolização é consideravelmente aumentada, ca $66 \%$ na primeira hora, evidenciando que o metabolismo in vivo de (1) é estritamente dependente do $\mathrm{pH}$ da biofase ${ }^{16}$.

\section{QUIRALIDADE}

A constatação no início da década de 60 dos efeitos teratogênicos provocados pela ( \pm )-talidomida em gestantes, nos três primeiros meses de gravidez ${ }^{7,8}$, representou um marco na conscientização do risco da administração de um fármaco em sua forma racêmica, quando a razão eudísmica entre os dois enantiômeros é desconhecida (Figura 2).

Posterior tentativa de resolução cromatográfica de (1) e administração das espécies enantiomericamente puras, i.e., $(R) \mathrm{e}$ (S)-(1), demonstrou que o efeito teratogênico da talidomida era proveniente do emprego do enantiômero de configuração absoluta $(S)$, enquanto seu antípoda era desprovido de ação teratogênica ${ }^{17}$. Todavia, a estereoespecificidade da ação teratogênica de (1) foi evidenciada como sendo dependente da espécie animal

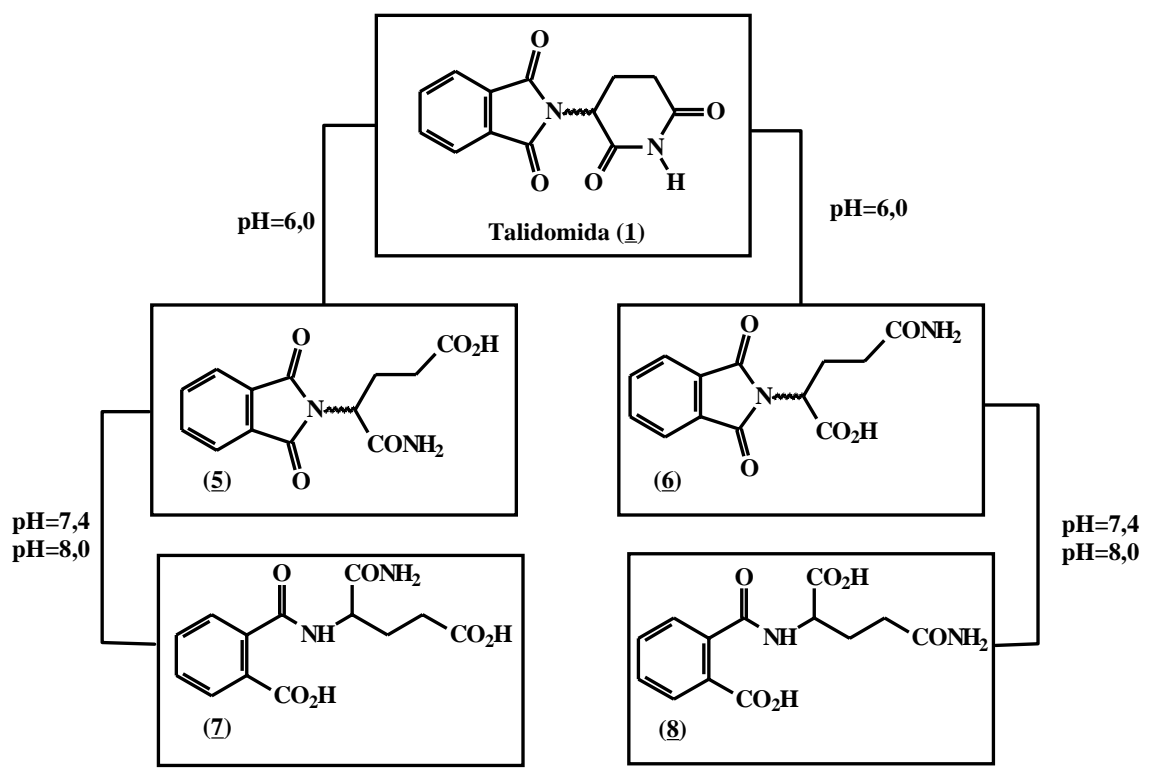

Figura 1. Principais metabólitos in vivo da talidomida (1).

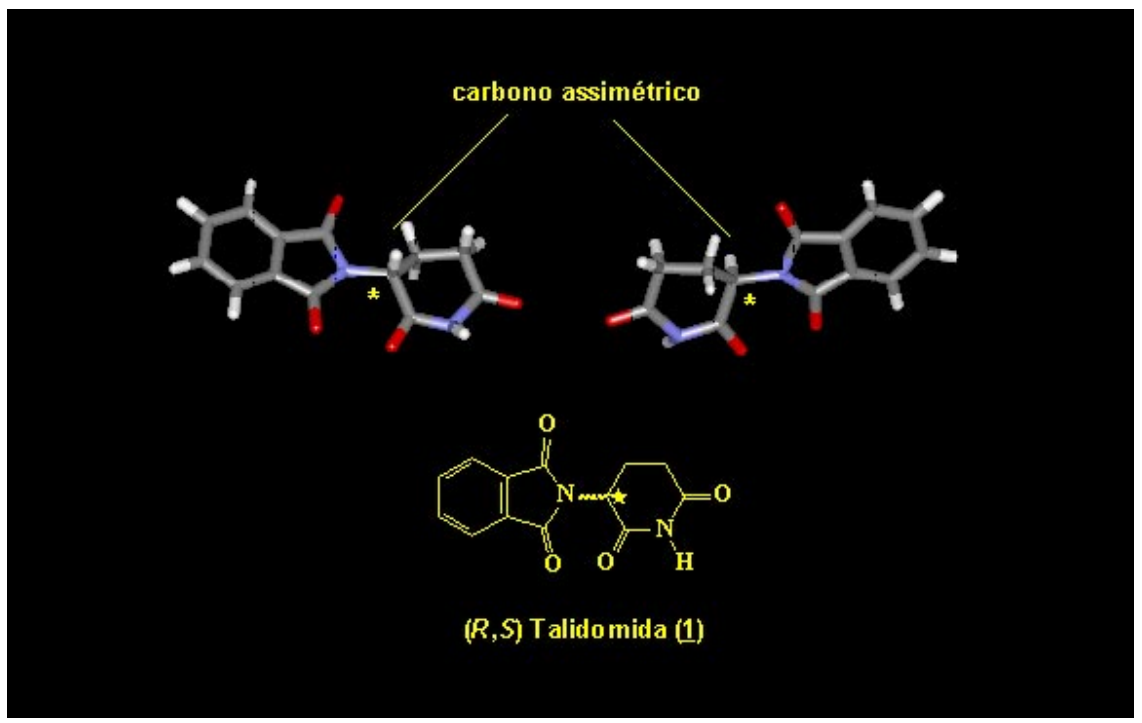

Figura 2. Demonstração esquemática dos enantiômeros da talidomida (1). 
estudada. Por outro lado, a atividade de (1) como inibidor da produção de TNF- $\alpha$ foi identificada como sendo estereosseletiva, tendo-se o enantiômero de configuração absoluta $(S)$ como eutômero $^{17,18}$.

Eriksson e colaboradores demonstraram a rápida epimerização de (1) em plasma humano, in vivo, após administração por via oral das espécies enantiomericamente puras. De fato, a inversão de configuração de fármacos enantiopuros ou racêmicos ocorre em outras classes terapêuticas como nos antiinflamatórios não-esteróides pertencentes à classe dos ácidos 2-aril-propiônicos ${ }^{20}$, sugerindo que o emprego da talidomida em sua forma enantiomericamente pura não evitaria a tragédia teratogênica ocorrida no início da década de $60^{18}$. Em trabalho mais recente, estes autores relataram estudos comparativos sobre a inversão de quiralidade e hidrólise de (1), em pacientes tratados, administração por via oral, com talidomida $(1)^{19}$. Os resultados obitdos a partir destes estudos denotam uma nítida diferença farmacocinética quanto aos processos de absorção e eliminação dos enantiômeros de (1), e comparando-se os resultados obtidos in vitro versus in vivo sugere-se que o processo de epimerização seja dependente da concentração de albumina, predominando, portanto, no sangue ou em compartimentos extravasculares ricos neste tipo de proteína sérica ${ }^{19}$. Em contra partida, estes estudos demonstraram que a taxa de hidrólise da talidomida (1), tanto in vitro quanto in vivo, parece ser dependente meramente do $\mathrm{pH}$ do meio, conforme preconizado por Schumacher e colaboradores em $1965^{16,19}$.

\section{TERATOGENICIDADE}

O termo teratogenicidade provém do grego "teratos", que significa monstro. $O$ sentido original da palavra refere-se a malformações anatômicas macroscópicas, embora atualmente tenha se expandido sua definição para englobar anomalias mais sútis como atraso intrauterino e distúrbios bioquímicos e psicomotores.

Embora várias hipóteses tenham sido postuladas para explicar os efeitos teratogênicos da talidomida (1), o mecanismo bioquímico responsável pela resposta embriotóxica em humanos e cobaios permanece obscuro e contraditório ${ }^{21,22}$. A dificuldade de se concluir sobre o mecanismo de indução de teratogenicidade por (1) in vitro e in vivo é, entre outros fatores, reflexo da sua baixa solubilidade e instabilidade na biofase. Por outro lado, as distinções interespécies quanto aos processos farmacocinéticos de absorção, metabolização, bioativação e eliminação à despeito do comportamento diferenciado, dependente do modelo animal empregado, o que dificulta a extrapolação para humanos, dos processos farmacocinéticos, explicam as diferenças de sensibilidade aos seus efeitos teratogênicos ${ }^{16}$.

Dentre as diversas hipóteses relatadas na literatura para explicar o mecanismo de teratogenicidade da talidomida $(1)^{23-27}$, encontra-se a hipótese descrita por Arlen \& Wells, que sugere o envolvimento da enzima prostaglandina endoperóxido sintase (PGHS) na bioativação do grupamento toxicofórico de (1) (Figura 3) ${ }^{22}$. A PGHS é uma enzima bifuncional com atividades cicloxigenase e hidroperoxidase, que catalisa a formação do hidroperóxido de prostaglandina $\mathrm{G}_{2}\left(\mathrm{PGG}_{2}\right)$, a partir de ácido araquidônico, e a subsequente biorredução da PGG2 à prostaglandina $\mathrm{H}_{2}\left(\mathrm{PGH}_{2}\right)$, precursora de uma família de icosanóides com propriedades inflamatórias, hiperalgésicas e trombogênicas ${ }^{28}$.

Esta hipótese fundamenta-se na atividade catalítica hidroperoxidase da PGHS. É amparada em observações experimentais, evidenciando que o tratamento de cobaias com ácido acetilsalicílico (Aspirina ${ }^{\circledR}$ ), um conhecido inibidor pseudoirreversível da PGHS, é capaz de prevenir, in vivo, as manifestações dos efeitos teratogênicos de $(1)^{22}$.

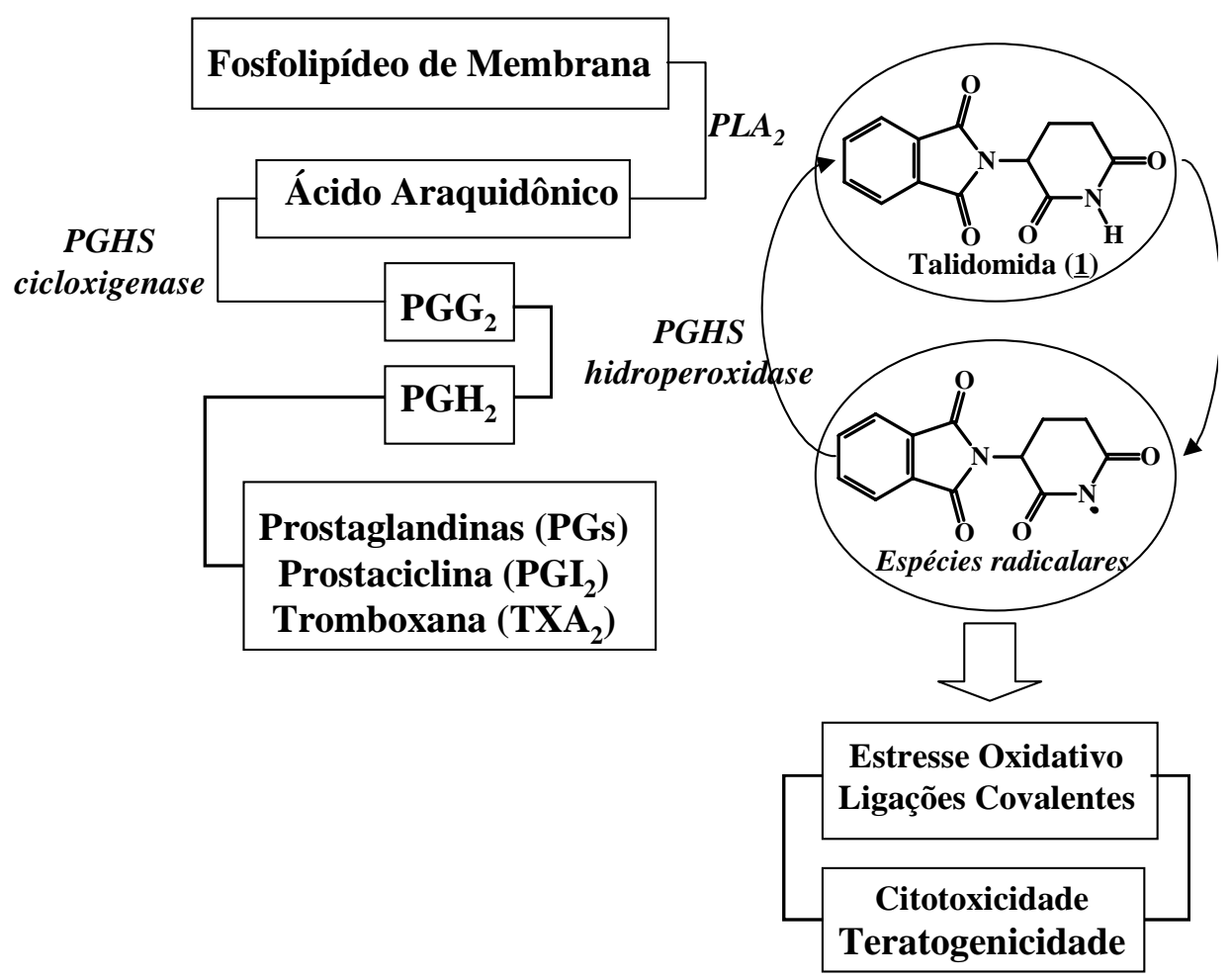

Figura 3. Representação ilustrativa da participação da PGHS na formação de espécies radicalares reativas de (1) (adaptado de Arlen \& Wells, 1996). 


\section{NOVAS APLICAÇÕES}

Com base na descoberta das propriedades antiinflamatórias e imunorreguladoras da talidomida (1), a indústria norte-americana Celgene Co. solicitou ao FDA sua aprovação para uso no tratamento da hanseníase, concedida em julho de 1998. Marcava-se de forma definitiva o renascimento deste fármaco, que representa, atualmente, um dos principais agentes terapêuticos disponíveis para o tratamento efetivo dos lepromas ${ }^{29-30}$

Após adquirir o direito de comercializar e estudar a talidomida (1), a Celgene Co. iniciou série de ensaios clínicos com este fármaco, visando ao tratamento de doenças auto-imunes relacionadas ao aumento da concentração plasmática de TNF- $\alpha$, tais como artrite reumatóide e doença de Crohn $n^{3,13,31-33}$. Trabalhos paralelos divulgados por vários laboratórios de pesquisa, demonstram a aplicação terapêutica potencial da talidomida no câncer, interferindo com o processo de angiogênese patológica, na AIDS, mais especificamente no retardamento da replicação do HIV, e no auxílio da perda de peso desordenada de portadores do vírus da imunodeficiência adquirida e do bacilo da tuberculose ${ }^{34-38}$. Adicionalmente, a talidomida representa a primeira terapia disponível nos últimos 20 anos para o tratamento de mieloma múltiplo ${ }^{4,39}$.

\section{ANÁLOGOS ESTRUTURAIS DA TALIDOMIDA}

Objetivando aprimorar-se as propriedades farmacocinéticas da talidomida (1) e reduzir seus efeitos teratogênicos, inúmeros esforços de pesquisa têm sido feitos na busca de análogos otimizados, com melhor perfil farmacoterapêutico, partindo-se da premissa de que se poderiam identificar e excluir os grupamentos toxicofóricos presentes em (1), resguardando-se os grupamentos farmacofóricos ${ }^{40,41}$.

A primeira abordagem do planejamento e síntese de novos análogos da talidomida (1) fundamentou-se em estudos prévios da relação entre a estrutura química e a atividade (SAR), visando identificar-se os grupamentos farmacofóricos necessários à atividade farmacológica pretendida. Com este intuito, modificações nas subunidades glutarimida e ftalimida foram realizadas e alguns exemplos de análogos estruturais de (1) planejados a partir destas modificações encontram-se ilustrados a seguir.

Shibata e colaboradores propuseram a síntese e a avaliação farmacológica de derivados $N$-alquil-ftalimídicos (9-12), planejados por modificação estrutural da subunidade glutarimida. Os resultados obtidos por estes autores demonstraram a irrelevância do grupamento glutarimídico na atividade antiTNF- $\alpha$ de (1) (Tabela 1$)^{42}$.

O mesmo grupo de pesquisadores, em trabalhos posteriores, demonstrou que a substituição do grupamento glutarimida por um anel fenílico reduzia a atividade anti-TNF- $\alpha$. Entretanto, a simples funcionalização na posição orto do anel fenila por grupos alquilas recuperava a atividade biológica desejada (Tabela 2 ) $^{43}$.

Em trabalho semelhante, Miyachi e colaboradores sintetizaram e avaliaram farmacologicamente derivados $N$-fenilftalimídicos funcionalizados (23-26), demonstrando que substituições nas posição orto, meta e para do anel fenila, por grupos alquilas, contribuem, de forma similar, para o aumento da atividade anti-TNF- $\alpha$ (Tabela 3$)^{44}$.

Com o objetivo de estudar a relevância farmacofórica do grupamento ftalimídico, Miyachi e colaboradores sintetizaram e avaliaram o perfil farmacológico dos derivados (27-31), constatando que, à exceção do composto tioimídico (28), modificações nesta subunidade são acompanhadas por decréscimo na atividade farmacológica (Tabela 4$)^{44}$.

Recentemente, os trabalhos de Muller e colaboradores demonstraram, reforçando os resultados descritos por Myiachi et. al. $(1997)^{44}$, a importância da presença do anel ftalimídico na atividade anti-TNF- $\alpha$ (Tabela 5$)^{45}$, sugerindo o caráter farmacofórico desta subunidade estrutural da talidomida (1).
Tabela 1. Atividade de derivados $\mathrm{N}$-alquil-ftalimídicos no bioensaio de produção de TNF- $\alpha$ por células HL-60, induzida por 12-O-tetradecanoilforbol-13-acetato (TPA)

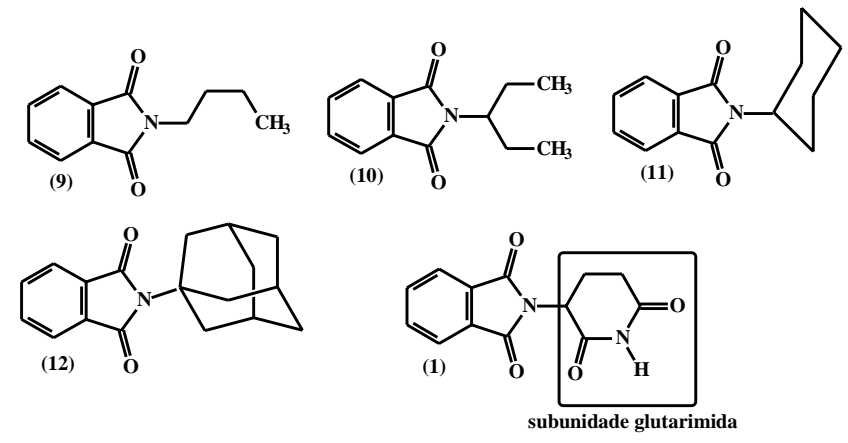

\begin{tabular}{cc}
\hline Composto $($ dose $=100$ ?M) & Quantidade de TNF- $\alpha(\%)^{\text {a) }}$ \\
\hline 9 & 102 \\
10 & 225 \\
11 & 182 \\
$\mathbf{1 2}$ & $\mathbf{5 2 1}$ \\
$\mathbf{1}$ & $\mathbf{1 5 5}$
\end{tabular}

a) A quantidade de TNF- $\alpha$ produzida na presença de $3 \mathrm{nM}$ de TPA foi definida como $100 \%$

Tabela 2. Atividade de derivados $\mathrm{N}$-fenil-ftalimídicos no bioensaio de produção de TNF- $\alpha$ por células HL-60, induzida por 12-O-tetradecanoilforbol-13-acetato (TPA)

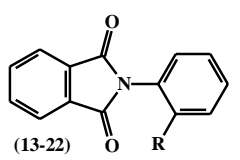

\begin{tabular}{ccc}
\hline $\begin{array}{c}\text { Composto } \\
(\text { dose }=100 \mu \mathrm{M})\end{array}$ & Substituinte $(\mathrm{R})$ & $\begin{array}{c}\text { Quantidade de } \\
\text { TNF- } \alpha(\%)^{\mathrm{a})}\end{array}$ \\
\hline 13 & $\mathrm{H}$ & 95 \\
14 & $\mathrm{CH}_{3}$ & 102 \\
15 & $\mathrm{C}_{2} \mathrm{H}_{5}$ & 116 \\
16 & $\left(\mathrm{CH}_{2}\right)_{2} \mathrm{CH}_{3}$ & 143 \\
$\mathbf{1 7}$ & $\left(\mathbf{C H}_{2}\right)_{3} \mathbf{C H}_{\mathbf{3}}$ & $\mathbf{1 6 4}$ \\
18 & $\left(\mathrm{CH}_{2}\right)_{4} \mathrm{CH}_{3}$ & 159 \\
19 & $\left(\mathrm{CH}_{2}\right)_{5} \mathrm{CH}_{3}$ & 141 \\
20 & $\left(\mathrm{CH}_{2}\right)_{6} \mathrm{CH}_{3}$ & 152 \\
21 & $\left(\mathrm{CH}_{2}\right)_{2} \mathrm{Ph}_{2}$ & 151 \\
$\mathbf{2 2}$ & $\mathbf{C H}\left(\mathbf{C H}_{3}\right)_{2}$ & $\mathbf{1 6 8}$ \\
\hline
\end{tabular}

${ }^{a}$ A quantidade de TNF- $\alpha$ produzida na presença de $3 \mathrm{nM}$ de TPA foi definida como $100 \%$

\section{CONCLUSÃO}

Quarenta anos após o incidente teratogênico da década de 60, a talidomida (1) ressurge como fármaco promissor para o tratamento da hanseníase, AIDS e tuberculose, levando esperança a pacientes desesperançosos. Entretanto, à despeito das novas aplicações terapêuticas da talidomida (1) seu emprego como fármaco requer avaliação da relação risco-benefício, exigindo em alguns casos monitoramento médico adequado.

Os relatos de SAR da talidomida e análogos revelam o potencial farmacofórico do anel ftalimídico ao mesmo tempo que denotam a irrelevância do grupamento glutarimida na atividade antiTNF- $\alpha$ mecanismo evidenciado como responsável pelos novos efeitos terapêuticos de (1). Estes dados, juntamente com hipótese que relaciona a teratogenicidade de (1) à ação enzimática da 
Tabela 3. Atividade de derivados $\mathrm{N}$-fenil-ftalimídicos no bioensaio de produção de TNF- $\alpha$ por células HL-60, induzida por 12-O-tetradecanoilforbol-13-acetato (TPA)

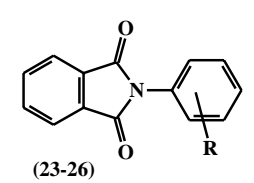

\begin{tabular}{ccc}
\hline $\begin{array}{c}\text { Composto } \\
(\text { dose }=30 \mu \mathrm{M})\end{array}$ & Substituinte (R) & $\begin{array}{c}\text { Quantidade de } \\
\text { TNF- } \alpha(\%)^{\mathrm{a})}\end{array}$ \\
\hline 23 & $\mathrm{H}$ & 100 \\
24 & $2-\mathrm{CH}\left(\mathrm{CH}_{3}\right)_{2}$ & 158 \\
25 & $3-\mathrm{CH}\left(\mathrm{CH}_{3}\right)_{2}$ & 158 \\
26 & $4-\mathrm{CH}\left(\mathrm{CH}_{3}\right)_{2}$ & 139 \\
\hline
\end{tabular}

a) A quantidade de TNF- $\alpha$ produzida na presença de $10 \mathrm{nM}$ de TPA foi definida como $100 \%$

Tabela 4. Perfil farmacológico de análogos da talidomida (1), apresentando variações na subunidade succinimida no bioensaio de produção de TNF- $\alpha$ por células HL-60, induzida por 12-Otetradecanoilforbol-13-acetato (TPA)
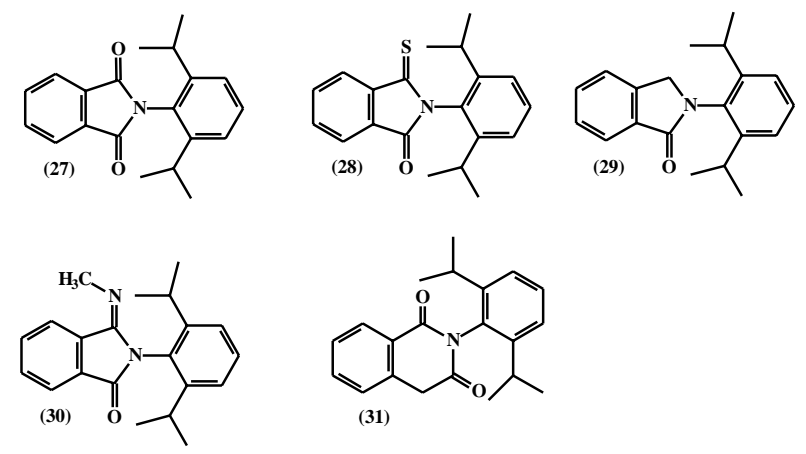

\begin{tabular}{cc}
\hline Composto $($ dose $=30 \mu \mathrm{M})$ & Quantidade de TNF- $\left.\alpha(\%) \mathrm{a}^{\mathrm{a}}\right)$ \\
\hline 27 & 602 \\
$\mathbf{2 8}$ & $\mathbf{1 2 5 2}$ \\
29 & 214 \\
30 & 134 \\
31 & 268
\end{tabular}

a) A quantidade de TNF- $\alpha$ produzida na presença de $10 \mathrm{nM}$ de TPA foi definida como $100 \%$

Tabela 5. Inibição da biossíntese de TNF- $\alpha$ estimulada por LPS em PBMC.
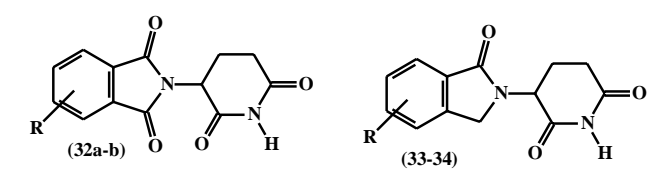

\begin{tabular}{cccc}
\hline Composto & Substituinte (R) & $\begin{array}{c}\text { Inibição da } \\
\text { produção de } \\
\text { TNF- } \alpha 100 \mu \mathrm{M}\end{array}$ & $\begin{array}{c}\text { Anti-TNF- } \alpha \\
\text { IC50 }\end{array}$ \\
\hline 32a & $\mathbf{( S ) - 4 - \mathbf { N H } _ { 2 }}$ & $\mathbf{9 9 \%}$ & $\mathbf{3 . 9 n M}$ \\
$\mathbf{3 2 b}$ & $\mathbf{( R ) - 4 - \mathbf { N H } _ { 2 }}$ & $\mathbf{8 5 \%}$ & $\mathbf{9 3 n M}$ \\
33 & $4-\mathrm{NH}_{2}$ & $74 \%$ & $100 \mathrm{nM}$ \\
34 & $5-\mathrm{NH}_{2}$ & $15 \%$ & ND \\
\hline
\end{tabular}

PGHS, denotam o perfil toxicofórico do grupamento glutarimida, viabilizando o planejamento e síntese de novos candidatos a fármacos, análogos à talidomida (1), com maior eficácia terapêutica e desprovidos de seu efeito teratogênico.

Vimos desenvolvendo no Laboratório de Avaliação e Síntese de Substâncias Bioativas (LASSBio) da Faculdade de Farmácia da Universidade Federal do Rio de Janeiro uma linha de pesquisa que visa à obtenção de novos análogos da talidomida, estruturalmente planejados de maneira a apresentar atividade antiinflamatória, modulando-se o perfil toxicofórico. Neste contexto, resultados recentes permitiram a obtenção de um composto ftalimídico com atividade antiinflamatória superior à talidomida (1), apresentando $\mathrm{ED}_{50}=2,5 \mathrm{mgKg}^{-1}$, e com expressiva atividade anti-TNF- $\alpha$ in vitro $46,47,48$.

\section{AGRADECIMENTOS}

Os autores agradecem as bolsas de doutorado e de produtividade do $\mathrm{CNPq}(\mathrm{Br})$ e aos assessores pelas sugestões que permitiram aprimorar a forma final deste manuscrito. Agradecemos a FUJB o pagamento de "page charges".

\section{GLOSSÁRIO}

Angiogênese: Crescimento de novos vasos sanguíneos.

Artrite reumatóide: Inflamação crônica de etiologia desconhecida associada a destruição progressiva de ossos e cartilagem.

Citocina: Glicopeptídeos biossintetizadas por vários tipos celulares possuindo múltiplas funções imunomoduladoras.

Grupamento farmacofórico: Grupo funcional ou grupos funcionais caracterizando subunidade estrutural responsável pela atividade farmacológica do fármaco.

Grupamento toxicofórico: Grupo funcional ou grupos funcionais caracterizando subunidade estrutural responsável pela toxicidade do fármaco.

Hanseníase: Doença infecciosa devida ao desenvolvimento no organismo do bacilo de Hansen, ie., Mycobacterium leprae, que penetra provavelmente pela pele ou pela mucosa nasal, e se propaga ao longo dos nervos periféricos.

Lepromas: Lesões características da hanseníase.

Lipopolisacarídeo (LPS): Componente da parede celular bacteriana capaz de estimular resposta inflamatória in vitro $e$ in vivo por meio de mecanismos envolvendo células do sistema imunológico.

Mieloma múltiplo: Tipo raro de câncer nos ossos.

PBMC (peripheral blood mononuclear cells): Mistura de células sanguíneas brancas, constituída primariamente de linfócitos e monócitos.

Razão eudísmica: Potência do eutômero (enantiômero com maior afinidade) relativa à do distômero (enantiômero de menor afinidade).

\section{REFERÊNCIAS}

1. Sneader, W.; Drug Discovery: The evolution of modern medicines 1985 John Wiley \& Sons, Inc., New York, pp.1.

2. Randall, T.; JAMA 1990, 263,1474.

3. Powell, R. J.; Brit. Med. J. 1996, 313, 377.

4. Raje, N.; Anderson, K.; N. Engl. J. Med. 1999, 341, 1606.

5. Mellin, G.W.; Katzenstein, M.; N. Engl. J. Med. 1962, 267, 1184.

6. Stirling, D.; Ann. Rept. Med. Chem. 1995, 30, 319.

7. McBride, W. G.; Lancet 1961, 1, 1358.

8. Lenz, W. Lancet 1962, 2, 45.

9. Cheymol, J.; Rev. Assoc. Med. Bra. 1965, 11, 123.

10. Kelsey, F. O.; Am. J.Public. Health 1965, 55, 703.

11. Koehler, C. S. W.; MDD 2000, 69. 
12. Sheskin, J.; Clin. Pharm. Ther. 1965, 6, 303.

13. Bessis, D.; Guillot, B.; Monpoint, S.; Dandurand, M.; Guilhou, J. J.; Lancet 1992, 339, 549.

14. Kaplan, G.; Sampaio, E. P.; Sarno, E. N.; Galily, R.; Cohn, Z. A.; J. Exp. Med. 1991, 173, 699.

15. Donnison, G. H.; U. S. Patent 1959, 2,876,225.

16. Schumacher, H.; Smith, R. L.; Williams, R. T.; Brit. J. Pharmacol. 1965, 25, 324.

17. Wnendt, S.; Zwingenberger, K.; Nature 1997, 385, 303.

18. Eriksson, T.; Björkman, S.; Roth, B.; Fyge, A.; Höglund, P.; Chirality 1995, 7, 44.

19. Eriksson, T.; Björkman, S.; Roth, B.; Fyge, A.; Höglund, P.; Chirality 1998, 10, 223.

20. Testa,B.; Carrupt, P. A.; Gal, J.; Chirality 1993, 5, 105.

21. Gordon, G. B.; Spielberg, S. P.; Blake, D. A.; Balasubramanian, V.; Proc. Natl. Acad. Sci. USA 1981, $78,2545$.

22. Arlen, R. R.; Wells, P. G.; J. Pharmacol. Exp. Ther. 1996, $277,1649$.

23. Tabin, C. J.; Nature 1998, 396, 322.

24. Neubert, R.; Merker, H -J.; Neubert, D.; Nature 1999, $400,419$.

25. Stephens, T. D.; Teratology 1988, 38, 229.

26. Neubert, R.; Hinz, N.; Thiel, R.; Neubert, D.; Life Sci. 1996, $58,295$.

27. Stephens, T. D.; Bunde, C. J. W.; Fuillmore, B. J.; Biochem. Pharmacol. 2000, 1489.

28. Mannett, L. J.; Environ. Health Perspect. 1990, 88, 5.

29. Calderon, P.; Anzilotti, M.; Phelps, R.; Int. J. Dermatol. 1997, 36, 881.

30. Aarestrup, F. M.; Sampaio, E. P.; de Moraes, M. O.; Albuquerque, E. C. A.; Castro, A. P. V.; Sarno, E. N.; Int. J. Leprosy. 2000, 68, 156.

31. Badger, A. M.; Adams, J. L.; Ann. Rept. Med. Chem. 1998, 33, 203 .

32. Hart, T.; Lamont, A.; Williams, D.; DDT 1998, 3, 516.

33. Sartor, R. B.; New Engl. J. Med. 2000, 1664.
34. James, J. S.; AIDS Treat News 1995, 3, 1.

35. Muller, G. W.; Chemtech 1997, 27, 21.

36. Rojas, M.; Olivier, M.; Gros, P.; Barrera, L. F.; García, L. F.; J. Immunol. 1999, 162, 6122.

37. Peuckmann, V.; Fisch, M.; Bruera, E.; Drugs 2000, 60, 273.

38. Kling, J.; $M D D$ 2000, 35.

39. Singhal, S.; Mehta, J.; Desikan, R.; Ayers, D.; Roberson, P.; Eddlemon, P.; Munshi, N.; Anaissie, E.; Wilson, C.; Dhodapkar, M.; Zeldis, J.; Barlogie, B.; N. Engl. J. Med. 1999, 341, 1565.

40. Corral, L. G.; Haslett, P. A. J.; Muller, G. W.; Chen, R.; Wong, L. -M.; Ocampo, C. J.; Patterson, R. T.; Stirling, D. A.; Kaplan, G.; J. Immunol. 1999, 163, 380.

41. Marriot, J. B.; Westby, M.; Cookson, S.; Guckian, M.; Goodbourn, S.; Muller, G.; Shire, M. G.; Stirling, D.; Dalgleish, A. G.; J. Immunol. 1998, 161, 4236.

42. Shibata, Y.; Shichita, M.; Sasaki, K.; Nishimura, K.; Hashimoto,Y.; Iwasaki, S.; Chem. Pharm. Bull. 1995, 43, 177.

43. Shibata, Y.; Sasaki, K.; Hashimoto, Y.; Iwasaki, S.; Chem. Pharm. Bull. 1996, 44, 156.

44. Miyachi, H.; Azuma, A.; Ogasawara, A; Uchimura, E.; Watanable, N.; Kobayashi, Y.; Kato, F.; Kato, M.; Hashimoto, Y.; J. Med. Chem. 1997, 40, 2858.

45. Muller, G. W.; Chen, R.; Huang, S. -Y; Corral, L. G.; Wong, L. M.; Patterson, R. T.; Chen, Y.; Kaplan, G.; Stirling, D. I.; Bioorg. Med. Chem. 1999, 9, 1625.

46. Lima, L. M.; Castro, P.; Fraga, C. A. M.; Gonçalves de Moraes, V. L.; Barreiro, E. J.; $23^{a}$ Reunião Anual da $S B Q$, Poços de Caldas, MG, 2000.

47. Lima, L. M.; Castro, P.; Fraga, C. A. M.; Claire, L.; Gonçalves de Moraes, V. L; Barreiro, E. J.; resultados não publicados.

48. Lima, L. M.; de Souza, S. D.; Menezes, C. M. S.; Castro, P.; Verli, H.; Fraga, C. A. M.; Rodrigues, C. R.; de Moraes, V. L. G.; Barreiro, E. J.; $24^{a}$ Reunião Anual da $S B Q$, Poços de Caldas, MG, 2001. 